\title{
Semiphenomenological spectral functions in neutrino scattering
}

\author{
Joanna E. Sobczyk* \\ Instituto de Física Corpuscular (centro mixto CSIC-UV), Institutos de Investigación de Paterna, \\ Apartado 22085, 46071, Valencia, Spain \\ E-mail: joanna.sobczykeific.uv.es

\section{Juan Nieves} \\ Instituto de Física Corpuscular (centro mixto CSIC-UV), Institutos de Investigación de Paterna, \\ Apartado 22085, 46071, Valencia, Spain \\ E-mail: jmnieveseific.uv.es
}

\begin{abstract}
We present a model for lepton-nucleus interaction which has been developed in a framework successully used to describe a variety of nuclear reactions. It is based on the local density approximation and accounts for some nuclear corrections needed to properly describe the processes induced by an electroweak probe.

We focus our analysis on the role played by the spectral functions which were obtained from a semiphenomenological model for the nucleon self energy inside of a nuclear medium [1]. The RPA (random phase approximation) effects are also considered in order to properly describe the collective excitations of the nuclei. At low energies the model has been compared with the data for muon and radiative pion capture processes. We also present results for the lepton-nucleus scattering cross section for a wide energy-momentum transfer range.
\end{abstract}

The 19th International Workshop on Neutrinos from Accelerators-NUFACT2017

25-30 September, 2017

Uppsala University, Uppsala, Sweden

${ }^{*}$ Speaker. 


\section{Introduction}

In the recent years the topic of lepton-nucleus interaction has been rediscovered in the context of neutrino physics. There is a tremendous activity aiming at achieveing an accurate theoretical description of neutrino-nucleus interaction to perform precise analyses of the neutrino properties.

We present a model for neutrino-nucleus interaction that has been successfully used to describe a variety of nuclear processes. It is based on the Local Density Approximation (LDA) which makes it appropriate to describe processes for different targets. Apart from the Pauli blocking, it incorporates two major nuclear effects: random phase approximation (RPA) and spectral functions (SFs). RPA describes the long-range nucleon-nucleon correlations, while spectral functions are responsible for the description of the nucleon's properties in the nuclear medium: the change of its dispersion relations and the appearance of a collisional broadening. We find an interplay of these two effects.

Lastly, we perform an extensive comparison of our model with data and results of other approaches for low energy processes such as muon and radiative pion capture, intermediate energy electron-nucleus scattering, and the neutrino scattering for both intermediate and high momentumenergy transfers.

\section{Theoretical framework}

The inclusive charge current cross section of neutrino scattering off a nucleus is given by

$$
\frac{d^{2} \sigma}{d \Omega d \omega}=\frac{|\vec{k}|}{\mid \vec{k}^{\prime}} \frac{G_{F}^{2}}{4 \pi^{2}} L_{\mu v} W^{\mu v}
$$

where $\vec{k}, \vec{k}^{\prime}$ are the incoming and outcoming lepton momenta, $L_{\mu v}$ and $W^{\mu v}$ are the leptonic and the hadron tensors. The tensor $W^{\mu v}$ is an object which encompasses all the physics of the hadron system. In our approach, we make use of the LDA, thus considering the interaction of a neutrino in the nuclear medium of a constant density and integrating it over the density profile of a given nucleus. Using Cutkosky rules, we determine $W^{\mu v}$ from the $W^{+}$self energy, $\Pi_{W}^{\mu v}$, in nuclear matter, as shown on the left panel of Fig. 1

$$
\begin{gathered}
W^{\mu v}(q)=W_{s}^{\mu v}(q)+i W_{a}^{\mu v}(q), \\
W_{s}^{\mu v}(q) \propto \int \frac{d^{3} r}{2 \pi} \operatorname{Im}\left(\Pi_{W}^{\mu v}+\Pi_{W}^{v \mu}\right), \\
W_{a}^{\mu v}(q) \propto \int \frac{d^{3} r}{2 \pi} \operatorname{Re}\left(\Pi_{W}^{\mu v}-\Pi_{W}^{v \mu}\right),
\end{gathered}
$$

where $q$ is the energy-momentum transfer. There is a great variety of nuclear processes contributing to the boson $W^{+}$self energy: $1 \mathrm{p} 1 \mathrm{~h}, 2 \mathrm{p} 2 \mathrm{~h}, \Delta$ excitation, etc. In what follows, we will focus on the quasielastic mechanism where the interaction takes place on one nucleon, producing another one (see the right panel of Fig. 1).

The particle-hole excitation $(1 \mathrm{p} 1 \mathrm{~h})$ contribution to $\Pi_{W}^{\mu v}$ can be expressed by

$$
-i \Pi_{W}^{\mu v}(q ; \rho)=-\cos ^{2} \theta_{C}\left(\frac{g}{2 \sqrt{2}}\right)^{2} \int \frac{d^{4} p}{(2 \pi)^{4}} G(p ; \rho) G(p+q ; \rho) A^{\mu v}(p, q),
$$



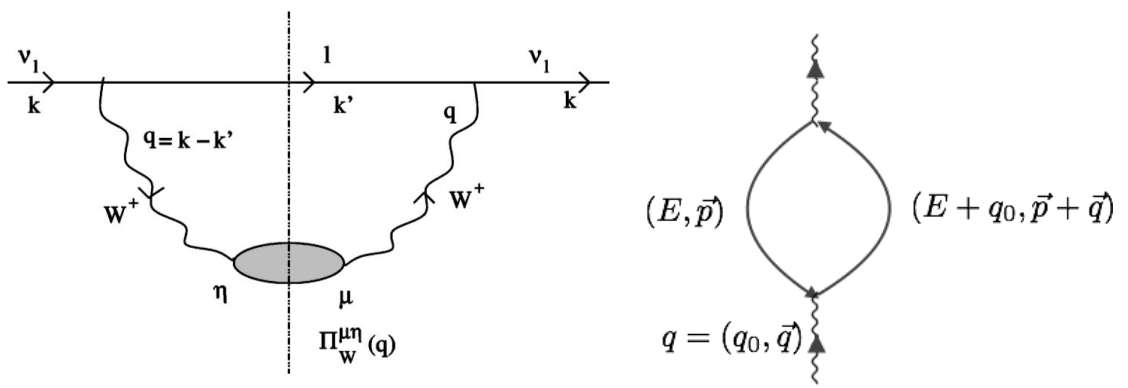

Figure 1: On the left: Neutrino $v_{l}$ traveling through the nuclear medium interacts via $\mathrm{W}^{+}$boson exhange producing a lepton $l$. The shade bubble stands for the $\mathrm{W}^{+}$self energy, $\Pi_{W}^{\mu \eta}$. The horizontal line shows a Cutkosky cut, placing the intermadiate particles on-shell. On the right: $1 \mathrm{p} 1 \mathrm{~h}$ contribution to the $\mathrm{W}^{+}$self energy.

where $G(p), G(p+q)$ are the in-medium Green's functions of a particle and a hole states, and $A^{\mu v}$ describes the vertices of interaction between the nucleons and the vector boson. In the Local Fermi gas (LFG), the Green's function describes a non interacting propagating state with an additional Pauli blocking factor.

It is convenient to introduce the $1 \mathrm{p} 1 \mathrm{~h}$ propagator (Lindhard function), that reads

$$
U(q, \rho)=-2 i \int \frac{d^{4} p}{(2 \pi)^{4}} 2 M G(p, \rho) 2 M G(p+q, \rho)
$$

The Lindhard function will be useful to perform an analysis of the spectral functions effect.

\section{Spectral functions and RPA}

Nucleons interact with each other in the nuclear medium, leading to an altered energy-momentum dispersion relation and a collisional broadening. These changes are encoded into the Green's function by means of the nucleon's self energy $\Sigma(E, \vec{p})$, where we omit the $\rho$ dependence:

$$
G(E, \vec{p})=\frac{1}{2 M} \frac{1}{E-M-\vec{p}^{2} / 2 M-\Sigma(E, \vec{p})}
$$

The hole and particle spectral functions are defined as:

$$
S_{h / p}(E, \vec{p})= \pm \frac{1}{\pi} \operatorname{Im} G(E, \vec{p})= \pm \frac{1}{\pi} \frac{\operatorname{Im} \Sigma(E, \vec{p})}{\left[E-\vec{p}^{2} / 2 M-\operatorname{Re} \Sigma(E, \vec{p})\right]^{2}+[\operatorname{Im} \Sigma(E, \vec{p})]^{2}}
$$

where $S_{h}(E, \vec{p})$ is defined for $E<\mu$ and $S_{p}(E, \vec{p})$ for $E>\mu ; \mu$ being the chemical potential. We use a model for nucleon self energy, $\Sigma(E, p)$, in the nuclear medium derived in [1]. It is calculated with a semiphenomenological approach which takes as an input the nucleon-nucleon elastic cross section and introduces some in-medium modifications by summing up some polarization diagrams.

The imaginary part of the Lindhard function and the hadron tensor can be expressed by means of the spectral functions [2]:

$$
\begin{gathered}
\operatorname{ImU}(q, \rho)=-\frac{\Theta\left(q^{0}\right)}{4 \pi^{2}} \int d^{3} p \int_{\mu-q^{0}}^{\mu} d \omega S_{h}(\omega, \vec{p}) S_{p}\left(q^{0}+\omega, \vec{p}+\vec{q}\right) \\
W^{\mu v}(q) \propto-\frac{\Theta\left(q^{0}\right)}{4 \pi^{2}} \int d^{3} r \int d^{3} p \int_{\mu-q^{0}}^{\mu} d \omega S_{h}(\omega, \vec{p}) S_{p}\left(q^{0}+\omega, \vec{p}+\vec{q}\right) A^{\mu v}(p, q)
\end{gathered}
$$



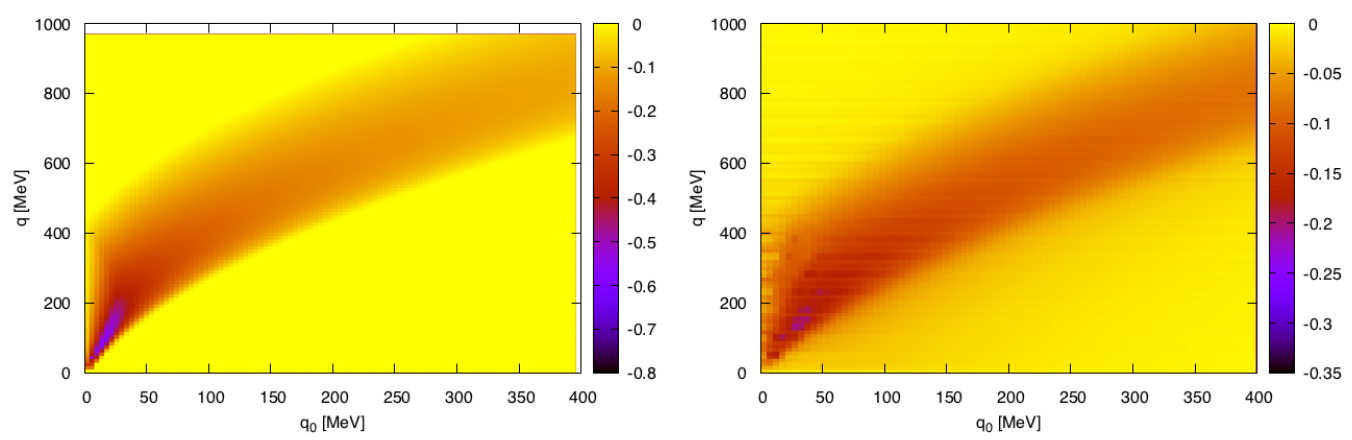

Figure 2: Imaginary part of the Lindhard function $\left|\operatorname{Im} U\left(q_{0},|\vec{q}|\right)\right|$ at the density $\rho=0.9 \mathrm{fm}^{-3}$. On the left, for non-interacting system, on the right, after introducing the spectral functions.

The effect of the spectral functions on $\operatorname{Im} U\left(q^{0}, \vec{q}\right)$ is shown on Fig. 2. Comparing the situation of the free LFG and an interacting system, we observe that the position of the quasielastic peak has been shifted (moving towards higher $q_{0}$ ) and quenched, so that $\operatorname{Im} U\left(q^{0}, \vec{q}\right) \neq 0$ for a much larger range of $\left(q^{0}, \vec{q}\right)$ values. These effects will be directly visible when analyzing the differential cross sections of various processes in the next section.

On top of the spectral functions, we include RPA effects which are responsible for the description of the collective degrees of freedom, especially important for the low energy-momentum transfer region. We introduce them into the model by summing the polarization diagrams which effectively amounts to a change of the strength of the interaction felt by the electroweak probe. With some caution (see the discussion in [2] Sec. II B), we will introduce the two effects together, bearing in mind that the RPA parameters were adjusted for the non interaction LFG system.

\section{Results and comparisons}

The model has been validated for a variety of lowenergy processes and also for intermediate-energy electron scattering where we have plenty of the data sets to compare with. Firstly, let us consider radiative pion and muon capture processes:

$$
\begin{aligned}
& \left(A_{Z}-\pi^{-}\right)_{\text {bound }}^{1 s} \rightarrow \gamma+X \\
& \left(A_{Z}-\mu^{-}\right)_{\text {bound }} \rightarrow \bar{v}+X
\end{aligned}
$$

where the initial nuclear systems are not stable because $\pi^{-}$ and $\mu^{-}$are much heavier than $e^{-}$and so their wavefunctions have a significant overlap with the nucleus. Making use of the LDA, we calculate the width for those processes.

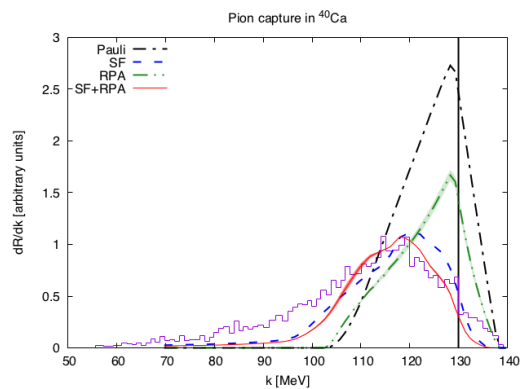

Figure 3: Results for the differential width $d \Gamma / d k$ for radiative pion capture. Data taken from [3].

The resulting prediction for the differential width for radiative pion capture from ${ }^{40} \mathrm{Ca}$ is shown in Fig. 3. We see that after introducing SFs and RPA 


\begin{tabular}{||l|cccc|c||}
\hline Nucleus & Pauli $\left(10^{4} \mathrm{~s}^{-1}\right)$ & RPA $\left(10^{4} \mathrm{~s}^{-1}\right)$ & $\mathrm{SF}\left(10^{4} \mathrm{~s}^{-1}\right)$ & $\mathrm{SF}+\mathrm{RPA}\left(10^{4} \mathrm{~s}^{-1}\right)$ & Exp. $\left(10^{4} \mathrm{~s}^{-1}\right)$ \\
\hline \hline${ }^{12} \mathrm{C}$ & 5.76 & $3.37 \pm 0.16$ & 3.22 & $3.19 \pm 0.06$ & $3.79 \pm 0.03$ \\
${ }^{16} \mathrm{O}$ & 18.7 & $10.9 \pm 0.4$ & 10.6 & $10.3 \pm 0.2$ & $10.24 \pm 0.06$ \\
${ }^{18} \mathrm{O}$ & 13.8 & $8.2 \pm 0.4$ & 7.0 & $8.7 \pm 0.1$ & $8.80 \pm 0.15$ \\
${ }^{23} \mathrm{Na}$ & 64.5 & $37.0 \pm 1.5$ & 30.9 & $34.3 \pm 0.4$ & $37.73 \pm 0.14$ \\
${ }^{40} \mathrm{Ca}$ & 498 & $272 \pm 11$ & 242 & $242 \pm 6$ & $252.5 \pm 0.6$ \\
\hline \hline
\end{tabular}

Table 1: Experimental and theoretical total muon capture widths for different nuclei. Data are taken from Ref. [4].

corrections, the peak of the distribution moves towards higher $\gamma$ momentum staying in agreement with the data. Also, the quenching of the peak and an appearance of a tail spreading to a higher momentum transfer is well visible. However, for higher momentum transfers we underestimate the data. This missing strength can be understood by means of $2 \mathrm{p} 2 \mathrm{~h}$ contributions that are not included in our calcuations.

For muon capture processes we may check our predictions with the experimental value of the total width for different nuclei (see Table 1). It is interesting to notice that the inclusion of the SFs and the RPA corrections do not change significantly the overall rate when compared to the inclusion of SFs alone.

Staying in the regime of low energy transfer, we also make predictions for $v-{ }^{12} \mathrm{C}$ inclusive cross sections integrated over neutrino flux, reported by LSND, LAMPF and KARMEN experiments (see Table. 2). As have been noticed previously, adding RPA on top of SFs does not change much the total cross section.

\begin{tabular}{||c|cc|cc|cc||}
\hline & Pauli & RPA & SF & SF+RPA & Experiment & \\
\hline & & & & & $11.2 \pm 0.3 \pm 1.8$ & LSND [5] \\
$\bar{\sigma}\left(v_{\mu}, \mu^{-}\right)$ & 23.1 & $13.2 \pm 0.7$ & \multirow{2}{*}{12.2} & $9.7 \pm 0.3$ & $8.3 \pm 0.7 \pm 1.6$ & LSND [6] \\
& & & & & $10.6 \pm 0.3 \pm 1.8$ & LSND [7] \\
\hline & & & & & $0.15 \pm 0.01$ & LSND [8] \\
$\bar{\sigma}\left(v_{e}, e^{-}\right)$ & 0.200 & $0.143 \pm 0.006$ & 0.086 & $0.138 \pm 0.004$ & $0.15 \pm 0.01 \pm 0.01$ & KARMEN [9] \\
& & & & & $0.141 \pm 0.023$ & LAMPF [10] \\
\hline
\end{tabular}

Table 2: Experimental and theoretical flux averaged ${ }^{12} \mathrm{C}\left(v_{\mu}, \mu^{-}\right) X$ and ${ }^{12} \mathrm{C}\left(v_{e}, e^{-}\right) X$ cross sections in $10^{-40}$ $\mathrm{cm}^{2}$ units.

At intermediate energy-momentum transfers, we test our model against the electron data. Owing to the fact that in this case the interaction has only a vector component (with the nucleon form factors under control) we may perform a precise analysis of the nuclear effects. We observe an excellent agreement with the data for kinematics where the relativistic effects are negligible (see the left panel of Fig. 4). The inclusion of the SFs quenches the peak and shifts it to the right position. For the higher energy transfer, however, one can see (Fig. 4, right panel) that the predicted quasielastic peak is too broad due to the nonrelativistic kinematics employed. In fact, this problem might be circumvented by either neglecting the particle spectral function or by using its relativized form as shown in [11]. 

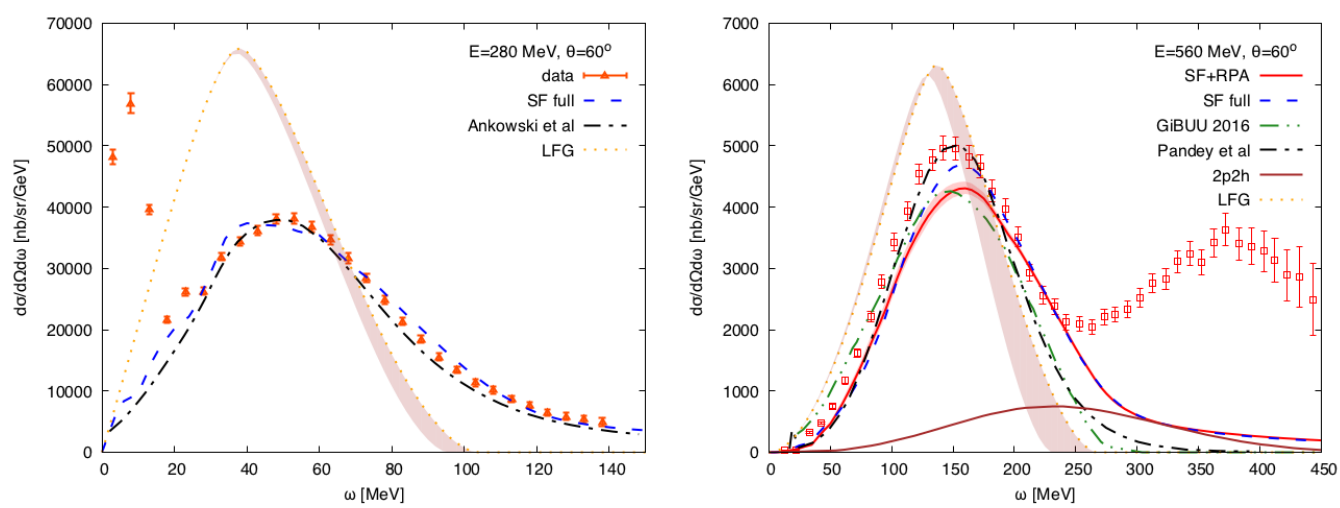

Figure 4: Electron scattering off ${ }^{12} C$ for two different kinematics. Bands for LFG curve show how the result changes when a relativistic kinematics is applied. Data taken from [12]. We also compare with Ankowski et al [13], GiBUU 2016 [14] and Pandey et al. [15].

Finally, in what concerns to the predictions for the neutrino-nucleus interaction, we want to stress that the inclusion of the nuclear effects is important even if we consider ratio $\sigma_{\mu} / \sigma_{e}$. The nuclear corrections do not cancel even though they diminish with the energy of incoming neutrino (see Fig. 5). In many neutrino experiments we have to deal with an energy transfer range beyond the applicability of the non relativistic kinematics. For this reason we also compare our predictions in the case when the spectral function is applied only to the hole state. In this approximation we get a reasonable results that compare rather well with those reported in [16] shown in Fig. 5. For a recent analysis of scaling properties and a comparison of our approach with [16], see [17].
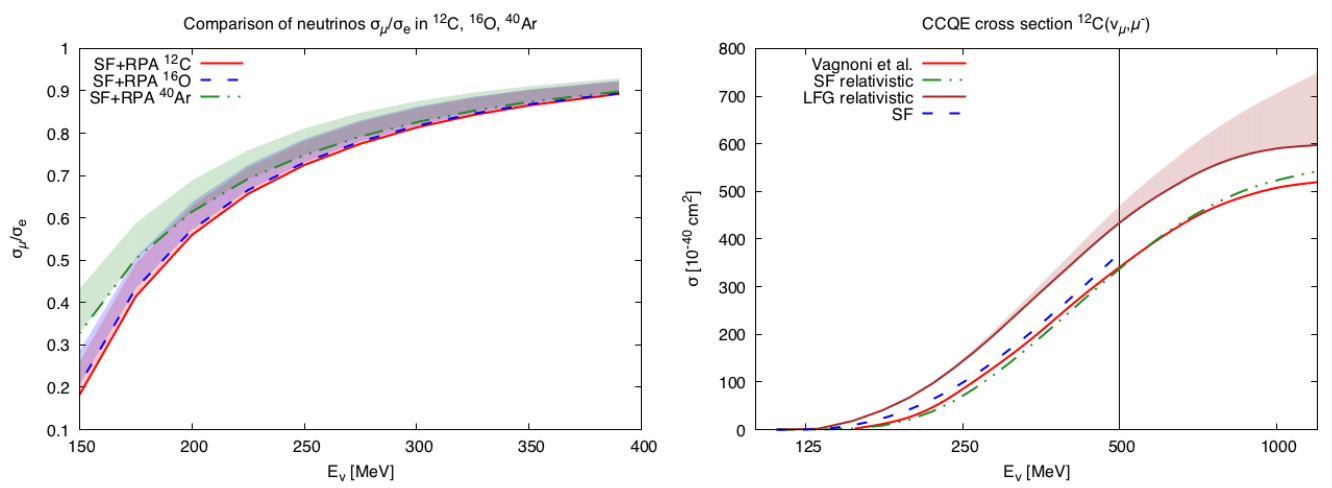

Figure 5: Predictions for neutrino-nucleus scattering. On the left: ratio of the total cross section for $v_{\mu}$ and $v_{e}$ for three targets. On the right: total cross section as a function of incoming neutrino. A band above LFG curve shows the effect of using a nonrelativistic kinematics. The predictions of our model are compared with those reported in Vagnoni et al. [16]

\section{Acknowledgement}

This research has been supported by the Spanish Ministerio de Economía y Competitividad and European FEDER funds under contracts FIS2014-51948-C2-1-P and SEV-2014-0398, by Generalitat Valenciana under Contract PROMETEOII/2014/0068. 


\section{References}

[1] P. Fernandez de Cordoba and E. Oset, Semiphenomenological approach to nucleon properties in nuclear matter, Phys. Rev. C46 (1992) 1697-1709.

[2] J. Nieves and J. E. Sobczyk, In medium dispersion relation effects in nuclear inclusive reactions at intermediate and low energies, Annals Phys. 383 (2017) 455-496, [1701.03628].

[3] J. A. Bistirlich, K. M. Crowe, A. S. L. Parsons, P. Skarek and P. Truoel, Photon spectra from radiative absorption of pions in nuclei, Phys. Rev. C5 (1972) 1867-1883.

[4] T. Suzuki, D. F. Measday and J. P. Roalsvig, Total Nuclear Capture Rates for Negative Muons, Phys. Rev. C35 (1987) 2212.

[5] LSND collaboration, M. Albert et al., Measurement of the reaction C-12 (muon-neutrino, mu-) X near threshold, Phys. Rev. C51 (1995) 1065-1069.

[6] LSND collaboration, C. Athanassopoulos et al., Measurements of the reactions C-12 (muon-neutrino, mu-) N-12 (g.s.) and C-12 (muon-neutrino, mu-) X, Phys. Rev. C56 (1997) 2806-2819.

[7] LSND collaboration, L. B. Auerbach et al., Measurements of charged current reactions of muon neutrinos on C-12, Phys. Rev. C66 (2002) 015501.

[8] LSND collaboration, C. Athanassopoulos et al., Measurements of the reactions $C-12$ (electron-neutrino, e-) N-12 (g.s.) and C-12 (electron-neutrino, e-) $N^{*-12, ~ P h y s . ~ R e v . ~ C 55 ~(1997) ~}$ 2078-2091.

[9] KARMEN collaboration, B. E. Bodmann et al., Neutrino interactions with carbon: Recent measurements and a new test of electron-neutrino, anti-muon-neutrino universality, Phys. Lett. $\mathbf{B 3 3 2}$ (1994) 251-257.

[10] D. A. Krakauer et al., Experimental study of neutrino absorption on carbon, Phys. Rev. C45 (1992) 2450-2463.

[11] P. Fernandez de Cordoba, E. Marco, H. Muther, E. Oset and A. Faessler, Deep inelastic lepton scattering in nuclei at $x>1$ and the nucleon spectral function, Nucl. Phys. A611 (1996) 514-538, [nucl-th/9511038].

[12] P. Barreau et al., Deep Inelastic electron Scattering from Carbon, Nucl. Phys. A402 (1983) 515-540.

[13] A. M. Ankowski, O. Benhar and M. Sakuda, Improving the accuracy of neutrino energy reconstruction in charged-current quasielastic scattering off nuclear targets, Phys. Rev. D91 (2015) 033005, [1404.5687].

[14] K. Gallmeister, U. Mosel and J. Weil, Neutrino-Induced Reactions on Nuclei, Phys. Rev. C94 (2016) 035502, [1605.09391].

[15] V. Pandey, N. Jachowicz, T. Van Cuyck, J. Ryckebusch and M. Martini, Low-energy excitations and quasielastic contribution to electron-nucleus and neutrino-nucleus scattering in the continuum random-phase approximation, Phys. Rev. C92 (2015) 024606, [1412.4624].

[16] E. Vagnoni, O. Benhar and D. Meloni, Inelastic Neutrino-Nucleus Interactions within the Spectral Function Formalism, Phys. Rev. Lett. 118 (2017) 142502, [1701.01718].

[17] J. E. Sobczyk, N. Rocco, A. Lovato and J. Nieves, Scaling within the Spectral Function approach, 1711.06697. 